\title{
Corpus
}

4 | 2005

Les corpus politiques : objet, méthode et contenu

\section{Positionnements énonciatifs dans les vœux présidentiels sous la cinquième République}

Analyse des marques personnelles par les méthodes de cooccurrence

Jean-Marc Leblanc et William Martinez

\section{CpenEdition}

\section{Journals}

Édition électronique

URL : http://journals.openedition.org/corpus/347

DOI : $10.4000 /$ corpus.347

ISSN : 1765-3126

Éditeur

Bases ; corpus et langage - UMR 6039

Édition imprimée

Date de publication : 1 décembre 2005

ISSN : 1638-9808

\section{Référence électronique}

Jean-Marc Leblanc et William Martinez, « Positionnements énonciatifs dans les vœux présidentiels

sous la cinquième République », Corpus [En ligne], 4 | 2005, mis en ligne le 01 septembre 2006, consulté le 08 septembre 2020. URL : http://journals.openedition.org/corpus/347 ; DOI : https:// doi.org/10.4000/corpus.347

Ce document a été généré automatiquement le 8 septembre 2020

(c) Tous droits réservés 


\title{
Positionnements énonciatifs dans les vœux présidentiels sous la cinquième République
}

Analyse des marques personnelles par les méthodes de cooccurrence

\author{
Jean-Marc Leblanc et William Martinez
}

Introduction

Le corpus Vœux regroupe 43 discours de vœux des présidents de la République française prononcés entre décembre 1959 et décembre 2001. Si les 42000 occurrences et 5200 formes qui composent le corpus sont inégalement réparties auprès des cinq locuteurs qui prennent la parole ${ }^{1}$, la cohérence de la situation de communication et la dimension chronologique et multi-locuteurs assurent à cette série textuelle une forte homogénéité qui se prête parfaitement à la démarche lexicométrique. Nous avons concentré nos analyses statistiques sur trois marques personnelles très fréquentes dans le discours : JE (344 occ.), VOUS (326 occ.) et NOUS (655 occ.). En effet, leur emploi évolue au fil du texte: à l'énonciation fortement personnalisée, centrée sur le JE de Giscard (JE, spécificité +E5), mais multipliant aussi les marques en direction des Français (VOUS, +E20), on opposera en premier lieu l'importante prise de distance produite chez le général de Gaulle par le rejet des marques de la première personne du singulier (JE, E16) et de la seconde du pluriel (VOUS, -E25), la prise en charge de l'énoncé étant assurée par un NOUS dont le référent est essentiellement la France (NOUS, +E2).

1. Le JE présidentiel

2 Dans un précédent article [Fiala \& Leblanc 2004] nous examinions le JE présidentiel réduit à sa forme graphique et explorions les réseaux de cooccurrents construits autour de cette forme structurante participant de la construction de l'ethos présidentiel. Différentes méthodes ${ }^{2}$ éprouvées et comparées nous ont permis de mettre en évidence plusieurs phénomènes essentiels. On se propose ici de revenir sur les expériences menées sur la première personne du singulier au moyen d'une analyse en tri croisé réalisée à l'aide du logiciel Alceste. On considère cette analyse comme une synthèse des expériences précédemment menées où nous avions confronté les résultats de plusieurs 
mesures cooccurrentielles. Nous la produisons ici en tant qu'aboutissement de cette série d'expérimentations (tableau 1).

Classe $2: 714$ U.C.E $(73 \%)$

\begin{tabular}{|c|c|c|c|}
\hline Forme réduite & KZiz & \multicolumn{2}{|c|}{ Fompre réduite Khi2 } \\
\hline *loc_dg & 52,38 & trouwter & 4,1 \\
\hline nous & 14,84 & ailleurs & 3,97 \\
\hline $\sec 170 \mathrm{t2} 2+$ & 10,6 & 1eut: & 3,97 \\
\hline atzuée_1963 & 10,31 & gueme+ & 3,93 \\
\hline devoir. & 8,67 & modernet & 3,73 \\
\hline antrée_1965 & 7,29 & solid+e & 3,61 \\
\hline atunée_1961 & 7,27 & rappott+ & 3,61 \\
\hline atunée_1968 & 6,24 & acquerit & 3,61 \\
\hline at+11é__ 1960 & 6,16 & $50 \mathrm{i}$ & 3,61 \\
\hline moyent & 5,56 & que1 & 3,61 \\
\hline developpement+ & 5,5 & troubl+er & 3,61 \\
\hline *antrée_1966 & 5,12 & scientific & 3,61 \\
\hline armet & 5,07 & ensemble+ & 3,55 \\
\hline cooperati $+\mathrm{f}$ & 5,07 & *année_2000 & 3,55 \\
\hline tedtunt & 5,07 & 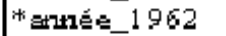 & 3,44 \\
\hline peuplet & 5,06 & mandiat & 3,44 \\
\hline facet & 4,71 & Inoment+ & 3,38 \\
\hline arganisat+ian & 4,71 & cas & 3,24 \\
\hline *atutée_2001 & 4,57 & dehors & 3,24 \\
\hline $\operatorname{soct15}$ & 4,52 & telle & 3,24 \\
\hline elle & 4,46 & mul tipliter & 3,24 \\
\hline jusqu+ & 4,34 & monetairet & 3,24 \\
\hline plant & & butt & 3,24 \\
\hline conflit+ & 4,34 & route+ & 3,24 \\
\hline inflatione & 4,34 & aidter & 3,01 \\
\hline
\end{tabular}


Tableau 1: Croisement de la variable JE avec l'ensemble du corpus (50 premières formes)

\begin{tabular}{|c|c|c|c|}
\hline \multicolumn{4}{|c|}{ Classe 1: 255 U.C.E (27\%) } \\
\hline Forme réduite & Khi2 & Forme réduite & Khiv \\
\hline ie & 969 & votre & 12,07 \\
\hline wous & 148,97 & soirt & \\
\hline souhgiter & 148,06 & bonne+ & 11,34 \\
\hline is & 60 & redire. & 11,25 \\
\hline dire. & 45,77 & tiens & 11,25 \\
\hline mes & 44,38 & present+er & 11,25 \\
\hline woent & 38,95 & echangter & 11,25 \\
\hline$x e+$ & 38,14 & metro & 10,98 \\
\hline form & 37,03 & savoir. & 10,9 \\
\hline & 27,54 & $\mathrm{~mm}$ & 10,52 \\
\hline antreet & 27,44 & soireet & 10,12 \\
\hline & 27,02 & $\mathrm{ava}$ & 10,12 \\
\hline heurteux & 23,87 & coeurt & 9,97 \\
\hline iote+ + & 23,61 & prom & 9,85 \\
\hline francaist & 23,31 & $p=r+1+e r$ & 9,43 \\
\hline adtess+ar & 22,45 & at+11ée_1974 & 9,34 \\
\hline cher- & 19,82 & $\operatorname{mon}$ & 9,34 \\
\hline $\mathrm{ai}$ & 18,98 & pas & 9,32 \\
\hline $\mathrm{m}+$ & 17,59 & ce & 9,27 \\
\hline & & tna & 8,67 \\
\hline & 14,12 & & 8,67 \\
\hline enjeut & 14,07 & recu+ & 8,43 \\
\hline adres set & 13,15 & sim+er & 8,43 \\
\hline *1ac_giscard & 12,76 & denand+er & 8,09 \\
\hline proposter & 12,36 & treppelter & 7,63 \\
\hline
\end{tabular}

Guide de lecture du tableau 1

L'analyse en tri croisé - ici sur la forme JE - consiste à croiser le pôle considéré à l'ensemble du corpus opposant ainsi deux classes d'énoncés formées en fonction de la présence ou de l'absence de la forme pôle. Contrairement à la procédure classique qui repose sur le principe de la classification descendante, $100 \%$ des U.C.E (unités de contexte élémentaire) sont classées. La totalité des U.C.E est prise en compte, sur la base d'une classification ascendante. Le tableau produit les formes réduites les plus significatives des deux classes obtenues, ordonnées selon le Khi2 décroissant, c'est-à-dire l'indice d'association à la classe.

On constate en premier lieu que le JE présidentiel est fortement conditionné par le rituel. Nous nous sommes livré dans notre recherche doctorale à une série d'expériences visant à faire abstraction de ce rituel lors du calcul des cooccurrents par la rétention d'une liste de formes, par la modification des seuils de fréquence et de probabilités, par l'établissement d'une liste créée à partir des classifications d'Alceste. Mais, nous avons également étudié les positionnements énonciatifs des cinq présidents de la République au sein des différentes thématiques du corpus afin d'examiner les variations énonciatives liées aux thèmes développés dans les allocutions. Ainsi note-ton parmi les premiers cooccurrents de nombreux verbes de circonstance (souhaiter, former), des formules d'adresse, un important champ lexical du rituel. La thématique des vœux est précisément très représentée dans la première classe, à travers des substantifs (vœux, année, soirée), à travers des adjectifs (heureux, bonne), mais aussi des verbes (adresser, présenter, échanger). Le rituel et les formules d'adresse sont par ailleurs constitutifs de cette première classe (compatriote +, cher +, métropole + ).

On s'aperçoit aussi que le JE des messages de vœux est fortement associé au référent de l'interlocuteur ainsi que nous l'avons repéré au moyen d'approches cooccurrentielles classiques où la seconde personne du pluriel était toujours dotée d'un fort indice de spécificité et donc placée parmi les premiers rangs des cooccurrents de $\mathrm{JE}^{3}$. On le constate ici encore puisque le VOUS est la forme la plus contributive des énoncés 
attestant la première personne du singulier. Cette configuration énonciative est très largement le fait du rituel (je vous souhaite...), mais pas uniquement. Chez Pompidou en particulier, la co-présence JE/VOUS crée un échange fort avec l'interlocuteur (je voudrais que vous compreniez, je voudrais que vous sachiez). Ainsi on mesure la forte teneur argumentative de ces propos lorsque l'on s'intéresse au contexte politique de ces réalisations où il s'agit ni plus ni moins pour le président de la République de démontrer le bien-fondé de son action politique ainsi que nous pouvons en juger dans les exemples suivants :

«[...] Je voudrais que vous compreniez, qu'en Angleterre, en Hollande, en Belgique, en Suisse, en Italie et même en Allemagne, la hausse des prix est, à l'heure actuelle, aussi rapide ou plus rapide que chez nous. c'est la preuve qu'il s'agit d'un phénomène international profond, auquel nous ne pouvons pas échapper [...]» (Pompidou, 1972).

«Je voudrais que cette certitude vous détourne du découragement ou de la résignation, et vous ouvre les voies de l'espérance et de la volonté. »(V.G.E, 1973).

5 Une analyse plus approfondie indique que la forme voudrais est intimement liée au référent de l'interlocuteur et sur-employée chez les locuteurs qui précisément multiplient les marques énonciatives en direction des Français. La valeur n'est donc que rarement purement volitive, les emplois étant essentiellement métadiscursifs et intervenant dans des annonces de plan où bien souvent le locuteur s'adresse à une certaine catégorie de Français (Je voudrais d'abord exprimer ma sympathie à toutes celles et à tous ceux qui vivent ces derniers jours de 1999 dans l'épreuve. [Chirac, 1999]). Giscard et Pompidou qui entretiennent un lien très étroit avec les Français emploient cette forme dans une modalité directive qui intensifie la relation (Je voudrais que vous sentiez, que vous compreniez...).

6 Les contextes de (je) veux, quant à eux, montrent une tendance vers des emplois métadiscursifs ou explicatifs (je veux dire), même si la volition apparaît parfois chez Chirac et Mitterrand dans une faible mesure.

7 On constate par ailleurs que le JE rejette le NOUS qui est en sous-emploi significatif ${ }^{4}$. Ainsi on s'interroge en ces termes : la dimension politique voire polémique de l'énoncé est-elle portée par le NOUS tandis que le JE est essentiellement mobilisé par le rituel ? Quelques éléments de réponse nous sont d'ores et déjà fournis par l'analyse en tri croisé puisque l'on peut observer un lexique plus intimiste, plus affectif dans la première classe, tandis que la classe 2 recense des formes relevant du domaine politique, économique et social (économie, développement, organisation, conflit, ...).

8 Par rapport à l'approche cooccurrentielle classique, l'analyse en tri croisé nous permet de situer également les locuteurs dans une approche synthétique. Ainsi le locuteur Giscard se révèle particulièrement contributif des énoncés où la première personne du singulier et le rituel sont fortement représentés, l'année 1974 nécessitant une attention particulière. A l'opposé, dans le camp du NOUS, de Gaulle serait le plus représentatif, les années 1963, 1965, 1961, 1968 et 1960, puis 1966 étant très fortement contributives. Cependant, d'autres locuteurs - en l'occurrence Chirac - seraient à la fois, plus ponctuellement, proches du NOUS et/ou des thématiques qui y sont associées (2000, 2001).

9 La lemmatisation ${ }^{5}$, mais aussi l'algorithme mobilisé, permettent de voir « remonter » des formes réduites qui ne nous seraient pas nécessairement apparues aussi clairement 
au moyen d'autres approches. C'est le cas dans la première classe des formes proposer, promettre et demander qui n'entrent pas directement dans le rituel des vœux.

En revanche, savoir, du fait de la réduction opérée est moins contributif que ne l'est la forme sais ${ }^{6}$ comme on peut le vérifier par exemple par analyse cooccurrentielle. La présence de ce cognitif n'est pourtant pas un fait négligeable. En termes de distribution statistique, il est d'avantage employé chez Chirac, et ses emplois chez tous les locuteurs (excepté de Gaulle pour qui la forme est absente) sont avant tout des renforçateurs d'empathie, mais également bien souvent argumentatifs. Cette marque d'empathie introduit dans de nombreux cas chez Chirac une relance incitative et mobilisatrice. Parmi les cooccurrents de la forme sais, on note une forte présence du VOUS de l'interlocuteur. Par ailleurs, sais participe de la construction d'un ethos de l'omniscience, de la clairvoyance. Ceci nous amène à engager la réflexion suivante: peut on repérer d'autres marques de l'empathie et de la compassion dans les messages de vœux? D'autres emplois des cognitifs sont-ils notables? (J'ai conscience, je vois, je comprends...). Nous pouvons déjà répondre partiellement à cette deuxième partie de la question: Chirac dans un discours volontariste et mobilisateur est celui chez qui les formes verbales sont attestées en plus grand nombre. Parmi celles-ci on trouve des verbes exprimant la conscience et la connaissance, visant à faire l'éloge des Français en les mettant en scène (Partout je vois des exemples de solidarité...).

\section{L'échange JE/VOUS}

11 Il n'est pas inintéressant de se pencher sur les phrases où les deux pôles énonciatifs JE/ VOUS sont attestés. Bien que l'immense majorité des contextes soit le fait de formules rituelles de présentation de vœux, on distingue à des fréquences moindres, quelques contextes où l'énonciation n'est plus simplement rituelle. Une connivence, un échange s'établissent selon des modalités différentes. Dans un premier cas de figure, l'interlocuteur est associé à la réflexion. C'est alors l'ethos du guide qui se construit au travers de ces emplois, mais d'un guide qui explique, explicite, clarifie son action et son discours. Il n'est d'ailleurs pas inattendu que de Gaulle n'apparaisse pas parmi de tels exemples :

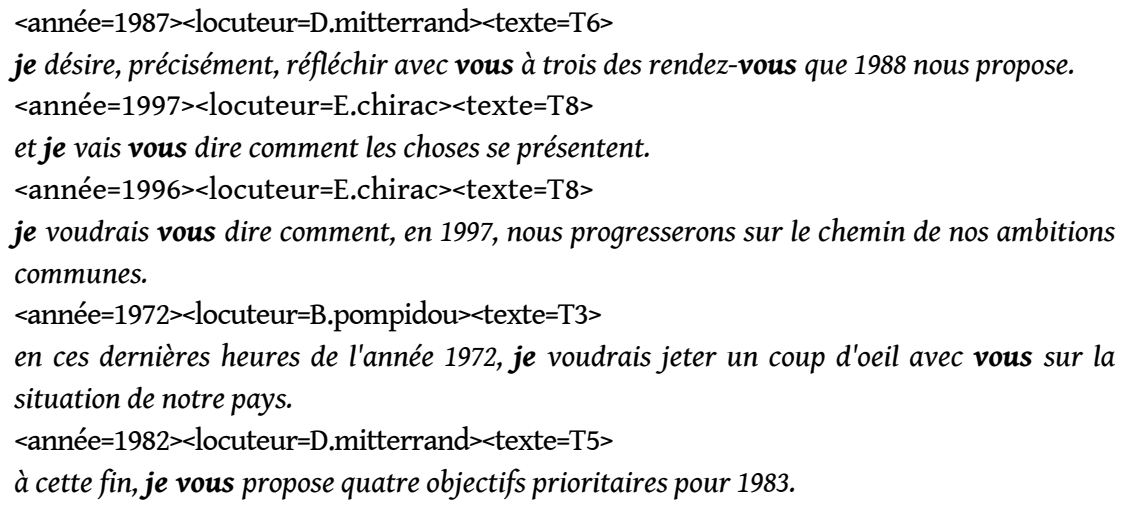

12 Cette connivence peut aussi naître de l'explicitation de l'énonciation, qui renforce l'effet de la fonction phatique tout en produisant l'insistance :

$<$ année=1980 $><$ locuteur=C.giscard $><$ texte=T5>

mais je vous dis bien haut que ce sont les plus françaises.

$<$ année $=1980><$ locuteur $=$ C.giscard $><$ texte=T5>

je vous le répète, j'ai confiance dans le progrès de la France.

$<$ année $=1979><$ locuteur $=$ C.giscard $><$ texte=T5>

mes chers amis, je reviens à mes mots du début pour vous exprimer mes vœux très affectueux pour 1980 . 
$<$ année $=1986><$ locuteur=D.mitterrand $><$ texte=T6>

mon autre souhait, je vous l'ai dit, est que la France sache vivre et faire vivre sa démocratie.

<année=1994><locuteur=D.mitterrand $><$ texte=T8>

je vous le dis avec la même passion que naguère.

13 Les deux pôles énonciatifs peuvent également s'articuler au sein d'un énoncé où la force illocutoire est celle de la promesse et de l'engagement. Le président engage alors sa confiance auprès des Français comme dans les exemples suivants :

<année=1971><locuteur=B.pompidou><texte=T3>

il maintiendra, je vous l'assure, la dignité de la France.

<année=1973><locuteur=B.pompidou><texte=T3>

mais je puis vous assurer que tout sera fait pour maintenir votre niveau de vie et votre pouvoir d'achat, notamment celui des plus faibles.

<année=1968><locuteur=A.degaulle><texte=T3>

un cœur que, depuis longtemps, permettez moi de le dire, n'épargnent pas les soucis au sujet du sort de la France, mais qui, je vous l'affirme, est aujourd'hui rempli d'espoir.

$<$ année $=1982><$ locuteur=D.mitterrand $><$ texte=T5>

quiconque est seul dans la vie, quiconque est pauvre, quiconque souffre d'être parmi les sans

travail, rencontrera, je vous l'assure, une société plus fraternelle.

$<$ année=1971 ><locuteur=B.pompidou><texte=T3>

il prendra, je vous le promets, les mesures indispensables au fil des circonstances, pour développer le bien-être et pour assurer un niveau convenable de l'emploi.

14 A un autre niveau c'est l'empathie qui est engendrée par la co-présence des deux personnes, le plus souvent associées à un verbe cognitif, ainsi que le montrent les contextes produits ci-dessous :

$<$ année $=1998><$ locuteur=E.chirac $><$ texte=T9>

je pense, comme vous, qu'il faut éviter ce qui divise inutilement, ce qui blesse les gens dans leurs convictions.

$<$ année=1972><locuteur=B.pompidou><texte=T3>

je suis convaincu que vous en avez conscience et c'est pourquoi c'est en pleine confiance et

de tout cœur que je vous dis ce soir : bonne année!

$<$ année $=2001><$ locuteur $=$ E. chirac $><$ texte $=$ T9 $>$

quelles que soient les épreuves récentes et les incertitudes de l'avenir, je sais que vous voulez faire vivre les valeurs qui sont celles de notre démocratie, de notre république.

$<$ année $=1980><$ locuteur $=$ C.giscard $><$ texte $=\mathrm{T} 5>$

lorsque l'usage de la liberté vous parait excessif - et je sais que beaucoup d'entre vous le pensent parfois - dites-vous que la liberté est un bien fragile, que tant d'autres hommes et tant d'autres femmes dans le monde voudraient connaître autant que nous, et qu'il nous faut savoir sauvegarder.

$<$ année $=2000<<$ locuteur=E.chirac $><$ texte=T9>

et vous avez, je le sais, de plus en plus ce désir de vous l'approprier, cette envie d'agir et d'avancer, cette soif de projets, de réalisations qui sont aujourd'hui les grands atouts de la France.

L'évocation du discours passé produit deux effets complémentaires. D'une part, elle a force de preuve et contribue à la constitution d'un ethos de la crédibilité, de l'omniscience, de la clairvoyance: les prédictions du chef de l'État se sont bien réalisées. D'autre part, elle tend à rapprocher les deux instances de l'énonciation : se rappeler ses propos c'est leur accorder une importance et accorder de l'importance à ceux à qui on les a tenus. Revenir sur son propre discours c'est aussi y mettre plus d'affectif et de subjectivité.

$<$ année=1992><locuteur=D.mitterrand $><$ texte=T7>

je vous en parlais ces dernières années, comme on parle d'une espérance. 
<année=1990><locuteur=D.mitterrand><texte=T7>

je me souviens de vous avoir déclaré, lors des premiers vœux que je vous adressais, le 31 décembre 1981: "tout ce qui permettra de sortir de Yalta sera bon", ce qui voulait dire «tout ce qui permettra d'en finir avec la division mortelle de l'Europe ».

<année=1971><locuteur=B.pompidou><texte=T3>

il y a un an, jour pour je vous disais: "nous ne sommes pas les plus forts, mais nous comptons et nous sommes respectés. "

$<$ année $=1973><$ locuteur=B.pompidou $><$ texte=T3>

Françaises, Français, il y a un an, en vous offrant mes vœux pour l'année 1973, je vous disais que ce serait une année d'expansion exceptionnelle et de grands progrès dans divers domaines.

<année=1971><locuteur=B.pompidou><texte=T3>

il y a un an, je vous disais encore: "nous ne sommes pas les plus riches, mais nous sommes parmi les plus heureux."

Une variation de ce mécanisme renforce particulièrement le caractère argumentatif chez Mitterrand qui établit sa légitimité en plaçant sa première allocution sous le signe du changement, et de l'histoire. Il articule sa démonstration autour d'une construction anaphorique dont les propositions centrales se font l'écho des engagements électoraux du candidat socialiste : « je vous avais promis », « nous l'avons fait ».

$<$ année $=1981><$ locuteur=D.mitterrand $><$ texte=T5>

je vous avais promis d'étendre le champ des libertés publiques.

$<$ année $=1981><$ locuteur=D.mitterrand $><$ texte=T5>

je vous avais promis d'entreprendre aussitôt les réformes qui permettraient ce changement.

<année=1981><locuteur=D.mitterrand><texte=T5>

je vous avais promis des réformes sociales.

$<$ année $=1981><$ locuteur=D.mitterrand $><$ texte=T5>

je vous avais promis de réduire la domination de l'état sur les individus, sur les collectivités

locales, communes, départements, régions.

Enfin, l'association JE/VOUS entre dans la réalisation de quelques « énoncés-appels » :

$<$ année $=1996><$ locuteur=E.chirac $><$ texte=T8>

je vous demande de rester vigilants.

$<$ année $=1960><$ locuteur=A.degaulle $>$ texte=T1>

Françaises, Français, je vous le demande, donnez au projet qui vous est soumis une

approbation immense.

$<$ année=1997 $><$ locuteur=E.chirac $><$ texte=T8>

je vous convie tous ardemment à mieux faire vivre notre démocratie.

$<$ année $=1976><$ locuteur $=$ C.giscard $><$ texte $=$ T4 $>$

françaises et français, mes contemporains et mes amis, je vous demande de prendre en charge avec moi, en 1977, le sort de la France, car ce sort ne dépend que de vous.

18 Sur ce point, il n'est pas inutile de rappeler les travaux de [Cotteret \& Moreau 1969] qui identifient dans les allocutions radiodiffusées du général de Gaulle deux groupes selon la proportion des marques personnelles qui y apparaissent. Les discours appels sont caractérisés par une forte densité du couple JE/VOUS, et les discours bilans par un « sur emploi » de la première personne du pluriel. Considérations dont il conviendrait de mesurer la pertinence -sur les autres locuteurs également - dans une approche plus détaillée de notre analyse. Cette distinction effectuée sur la base d'une distribution statistique, les auteurs constatent que des réseaux particuliers se forment autour de ces deux types discursifs. Autour des discours appels, caractérisés statistiquement par une dimension dialogique gravitent les formes République, État, Peuple, moi, Nation. Les discours bilans, articulés autour de la première personne du pluriel engendrent un champ lexical qui n'est pas sans rappeler le vocabulaire spécifique des vœux du général 
de Gaulle mais aussi le vocabulaire de la classe 2 que nous produisons ici : notre, année, économique, monde, développement, progrès.

3. Le NOUS présidentiel : approches cooccurrentielles du pronom

L'analyse des cooccurrences dans un corpus textuel repose sur le fondement que les mots ne sont pas des objets isolés les uns des autres mais qu'ils forment un système de relations syntaxiques et sémantiques qui leur donnent sens. En effectuant un appariement empirique des formes du corpus, la méthode présentée ici permet de détecter, au-delà des collocations strictement contiguës, des phénomènes d'attraction lexicale multiples et complexes afin de révéler une partie du réseau de cooccurrences qui régit le texte. Plus particulièrement cette approche peut s'avérer efficace pour résoudre une partie de l'ambiguïté référentielle du pronom NOUS.

3.1. De la cooccurrence à la poly-cooccurrence

Dans un premier temps nous avons pu associer successivement le pôle NOUS à différents cooccurrents suivant une mesure statistique qui met en évidence des associations lexicales deux à deux 7 . L'analyse détecte autour de NOUS un nombre modeste de 24 cooccurrents qui pour la plupart sont de spécificité moyenne ou faible (tableau 2) et dont un tiers sont des verbes. Cette prédication verbale se décline en trois types: les verbes statifs qui indiquent des propriétés ou des possessions dont les archilexèmes avons (+E51) et sommes (+E27), les verbes factifs qui transcrivent une action tels que faisons (+E06) et les verbes réflexifs déclaratifs qui transcrivent une déclaration sur un état ou une action tels que devons (+E08) et pouvons (+E06). Outre les mots-outils, surreprésentés dans l'environnement contextuel spécifique du pôle, on ne dénombre que deux noms autour de NOUS : liberté et moyens.

Tableau 2 : Cooccurrents positifs du pôle NOUS (contexte : phrase)

\begin{tabular}{|l|l|l|l|}
\hline forme & F & Co-fq & Spécif \\
\hline \hline avons & 103 & 103 & +E51 \\
\hline \hline sommes & 54 & 54 & +E27 \\
\hline \hline que & 677 & 282 & +E08 \\
\hline \hline chez & 28 & 23 & +E08 \\
\hline \hline devons & 15 & 15 & +E08 \\
\hline \hline allons & 14 & 13 & +E06 \\
\hline \hline faisons & 13 & 12 & +E06 \\
\hline \hline pouvons & 12 & 12 & +E06 \\
\hline \hline vivons & 11 & 11 & +E06 \\
\hline \hline nos & 166 & 78 & +E05 \\
\hline
\end{tabular}




\begin{tabular}{|l|l|l|l|}
\hline ensemble & 49 & 29 & $+\mathrm{E} 05$ \\
\hline \hline voulons & 12 & 11 & $+\mathrm{E} 05$ \\
\hline
\end{tabular}

\begin{tabular}{|l|l|l|l|}
\hline forme & F & Co-fq & Spécif \\
\hline \hline notre & 314 & 130 & +E04 \\
\hline \hline où & 78 & 39 & +E04 \\
\hline \hline en & 602 & 226 & + +E03 \\
\hline \hline dans & 351 & 138 & $+\mathrm{E} 03$ \\
\hline \hline plus & 269 & 107 & $+\mathrm{E} 03$ \\
\hline \hline si & 93 & 42 & $+\mathrm{E} 03$ \\
\hline \hline encore & 54 & 28 & $+\mathrm{E} 03$ \\
\hline \hline liberté & 39 & 20 & + +E03 \\
\hline \hline mêmes & 28 & 16 & $+\mathrm{E} 03$ \\
\hline \hline moyens & 24 & 14 & $+\mathrm{E} 03$ \\
\hline \hline commun & 13 & 9 & $+\mathrm{E} 03$ \\
\hline \hline construire & 13 & 9 & $+\mathrm{E} 03$ \\
\hline
\end{tabular}

Cette analyse cooccurrentielle ne livre donc dans un premier temps qu'une information élémentaire voire évidente sur les attractions lexicales du pôle NOUS, mais surtout, elle ne renseigne que sur des phénomènes de cooccurrence binaire alors que le texte est un objet disposé en différentes strates où se tissent de multiples interconnexions lexicales.

Bien sûr, on peut au vu de ces résultats hiérarchisés inférer des liens simultanés entre NOUS et plusieurs de ses cooccurrents pour établir des classes de mots dont les liens sont aisément validés par un retour au texte telles que /nous+voulons+construire/ ou / nous+avons+moyens/ (respecti-vement 3 et 4 réalisations en contexte). Toutefois, le problème d'interprétation des résultats demeure car le dépouillement lexicométrique du texte détruit son unité et fait disparaitre les liens inter-lexicaux. En effet, dès lors qu'un mot est extrait de la phrase qui constitue selon [Tesnière 1959] son « milieu naturel », il est "isolé comme dans un dictionnaire » et c'est rétrospectivement qu'il faut tenter de lui donner du sens. Alors, afin de saisir l'organisation interne du texte au-delà de ces cooccurrences binaires, il convient d'examiner plus précisément les relations cooccurrentielles multiples et simultanées qui unissent les mots les uns aux autres au sein de l'énoncé. 


\subsection{La méthode des poly-cooccurrences}

En lexicométrie les recherches s'orientent vers la détection automatique des associations lexicales complexes suivant deux principales méthodologies: l'approche lexico-graphique qui vise à produire des classes de mots à partir d'analyses quantitatives effectuées sur des corpus textuels (ex. : les lexicogrammes de [Heiden et Lafon 1998]) et l'approche morphosyntaxique qui s'applique à décrire la structure des phrases du texte (ex. : l'approche de [Habert et al. 1997]). Ici nous avons opté pour une approche purement lexicographique imaginée dans le prolongement de la méthode de cooccurrence présentée précédemment.

Il s'agit, après nous être interrogés sur les particularités cooccurrentielles du pôle NOUS, de nous intéresser aux formes caractéristiques des contextes du pronom lorsqu'il est accompagné d'un ou plusieurs cooccurrents donnés. Pour illustrer cette méthode le tableau 3 présente les cooccurrents détectés autour du pôle complexe noustavons. On découvre aussi que dans les 100 contextes où ces formes apparaissent simultanément, la forme raisons est surreprésentée. On réitère alors le calcul autour du pôle nous+avons+raisons pour constater que français en est un cooccurrent spécifique. La procédure exploratoire continue ainsi jusqu'à épuisement des cooccurrents ${ }^{8}$.

Tableau 3 : Poly-cooccurrents à partir du pôle NOUS (extrait) seuil de spécificité $\geq E 03$, contexte : phrase

\begin{tabular}{|l|l|l|l|l|l|}
\hline Pôle : nous+avons & & & & \\
\hline \hline 100 contextes (3 071 occurrences) & & & & \\
\hline forme & F & Co-Fq & Spéc. & Ctx \\
\hline droit & 27 & 8 & +E04 & 5 \\
\hline raisons & 11 & 5 & +E04 & 5 \\
\hline \hline strasbourg & 5 & 4 & +E04 & 1 \\
\hline \hline capitale & 3 & 3 & +E04 & 3 \\
\hline \hline pensons & 3 & 3 & +E04 & 1 \\
\hline
\end{tabular}

\begin{tabular}{|l|l|l|l|l|l|}
\hline Pôle : nous+avons+raisons & & & \\
\hline \hline 5 contextes (231 occurrences) & & & & \\
\hline \hline forme & & & & \\
\hline strasbourg & Co-Fq & Spéc. & Ctx \\
\hline \hline que & 677 & 4 & +E09 & 1 \\
\hline
\end{tabular}




\begin{tabular}{|l|l|l|l|l|}
\hline français & 142 & 4 & +E03 & 4 \\
\hline \hline parce & 45 & 3 & +E03 & 1 \\
\hline
\end{tabular}

Pour présenter les liens multiples et simultanés existant autour du pôle NOUS, l'analyse des réseaux de cooccurrences produit des résultats sous la forme de tableaux volumineux et denses : sur 466 poly-cooccurrences explorées, 95 sont jugées originales et répertoriées. Mais parce que la finalité d'une méthode des cooccurrences devrait être de proposer une vision planaire du réseau lexical, nous proposons dans une visée de cartographie lexicométrique une visualisation graphique qui facilite la lecture des réseaux produits par nos calculs. Dans la figure 1, on trouve un extrait du graphe plan qui représente le réseau de cooccurrences élaboré à partir du pôle NOUS réduit pour raison d'espace aux chemins de cooccurrences de spécificité $\geq$ E03. La lecture du graphe reproduit à l'identique le processus d'exploration contextuelle réitérée qui met au jour successivement de nouvelles cooccurrences spécifiques. On peut ainsi, en tout point du graphe, interpréter le parcours effectué grâce aux statistiques fournies pour chaque cooccurrent.

Guide de lecture du graphe de la figure 1

Le graphe se lit du haut vers le bas suivant la spécificité décroissante des cooccurrences détectées, et de la gauche vers la droite suivant l'expansion de chaque chemin de cooccurrence. Chaque nœud représente une forme et chaque arc indique la cooccurrence d'une forme nouvelle (à droite de l'arc) et d'une polycooccurrence déjà repérée (à gauche de l'arc). Pour faciliter la lecture des chemins de cooccurrence, chaque arc est codé par une cartouche à triple notation suivant la syntaxe suivante :

co-fréquence [spécificité de la cooccurrence] (contextes d'apparition)

Considérons sur le dessin le second chemin de cooccurrence, à savoir nous+avons+raisons+français. Le diagnostic de la cooccurrence avec la forme français, $4[+3](4)$, nous informe que cette forme apparaît 4 fois aux côtés du pôle nous et ses cooccurrents avons et raisons, avec une spécificité de +E03 et dans un total de 4 phrases. 
Figure 1 : Réseau de cooccurrences à partir du pôle NOUS (seuils de fréquence $\geq 3$ et de spécificité $\geq 3$, contexte phrase)

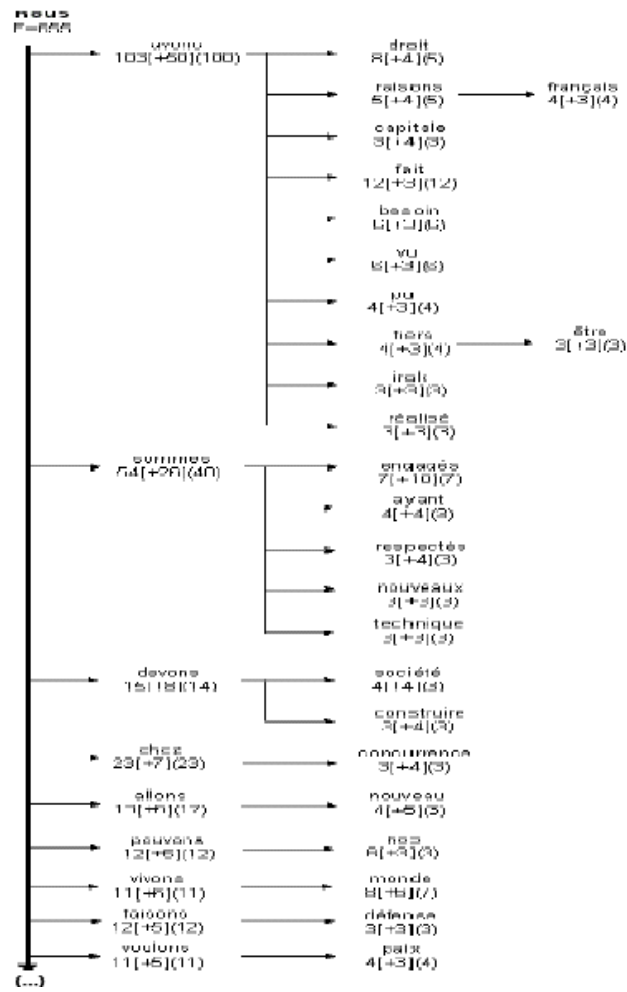

Figure 1 (suite) : Réseau de cooccurrences à partir du pôle NOUS seuils de fréquence $\geq 3$ et de spécificité $\geq 3$, contexte phrase)

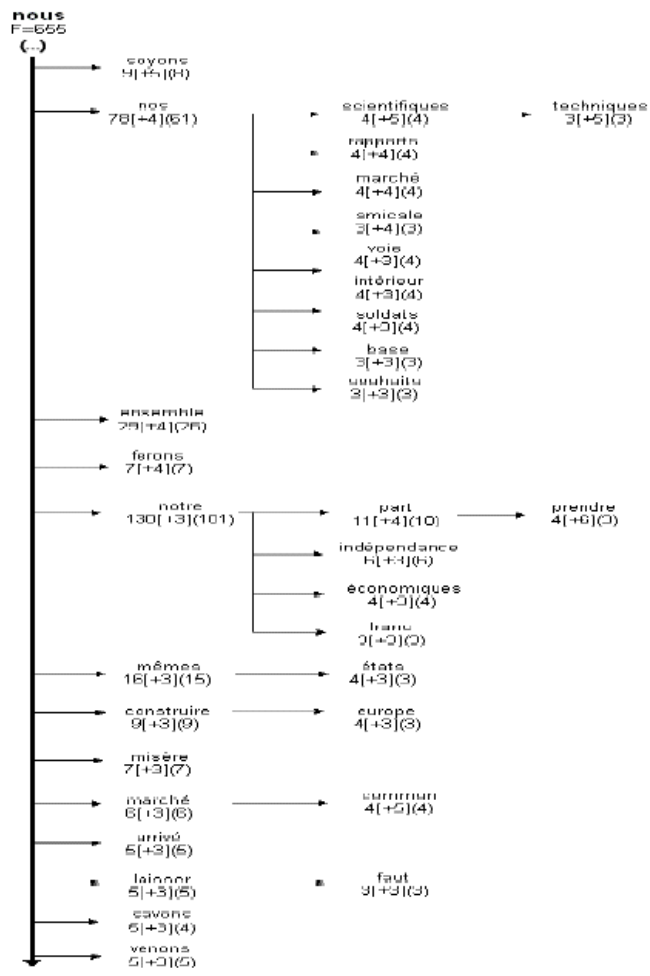



précisent différentes déclinaisons d'une même poly-cooccurrence, reflète une partie de la complexe ramification de l'activité cooccurrentielle interne du texte. En concentrant l'analyse autour du pôle NOUS pour explorer son environnement contextuel avec ce niveau de précision, on découvre au pronom des cooccurrents inédits. Ainsi, des noms et des adjectifs qui éludaient la méthode de cooccurrence initiale émergent désormais de ces réseaux complexes. Articulés autour des verbes, qui restent les cooccurrents les plus spécifiques du NOUS, apparaissent des prédicats nominaux (société, monde, marché, états, Europe, etc.) et adjectivaux (fiers, engagés, respectés, etc.). On note également que le vocabulaire des vœux, pourtant omniprésent dans le corpus, ne vient pas polluer les poly-cooccurrences détectées. parfaitement interprétables car il s'agit d'associations lexicales réelles dont on trouvera un nombre exact de manifestations effectives dans le texte. Cette approche écarte donc tout risque de créer selon l'expression de [Habert 1997] des «monstres langagiers " sans réalisation concrète et restitue une synthèse sans ambivalence des associations lexicales en contexte, ce qui diminue la part spéculative de l'interprétation des résultats. Ainsi on peut, muni de cette information cooccurrentielle, rechercher les contextes de réalisation de la poly-cooccurrence nous+avons+raisons +français :

$<$ année $=1965><$ locuteur=A.degaulle $><$ texte=T2>

enfin, notre expansion étant, à l'heure qu'il est, partie pour un bond en avant, nous avons toutes raisons de compter que dans les douze mois qui viennent, et comme l'indique notre plan, le niveau de vie des Français s'élèvera de 4\%.

$<$ année=1971>locuteur=B.pompidou><texte=T3>

avons-nous, en tant que Français, des raisons d'espérer?

$<$ année $=1972>$ locuteur=B.pompidou>texte=T3>

nous allons prochainement lancer un grand emprunt, dont je prédis par avance qu'il sera un succès, ce que jamais aucun gouvernement Français n'a tenté en période préélectorale et nous sommes persuadés et nous avons de bonnes raisons pour l'être que l'activité économique en 1973 sera très grande et qu'elle permettra de nouveaux progrès sociaux en faveur des plus défavorisés d'abord.

$<$ année $=1988>$ locuteur $=$ D.mitterrand $>$ texte $=$ T6 $>$

mes chers compatriotes, parce que c'est à Strasbourg que Rouget de l'Isle a, pour la première fois, chanté la marseillaise - le chant de la patrie et de la république - parce que Strasbourg est la capitale de l'Europe et que, cette Europe, nous avons quatre ans, pas davantage, pour la construire, parce que Strasbourg vient de fêter son deuxième millénaire, et pour bien d'autres raisons qui font que Strasbourg est aimée des Français, je suis heureux de vous présenter, ce soir et de cette ville, mes vœux de nouvel an.

Conclusion

L'approche cooccurrentielle des corpus et plus particulièrement l'approche polycooccurrentielle permettent une vue synoptique du texte à la lumière d'une unité discursive qu'il est impossible de comprendre à l'issue du dépouillement lexicométrique et qu'il est même parfois difficile de saisir dans le texte d'origine ! Entre les extrêmes de ces deux lectures opposées, la méthode poly-cooccurrentielle nous rappelle que les faits linguistiques sont soumis à des régularités qui régissent non pas des unités lexicales isolées mais des ensembles complexes de formes.

Sur le plan du discours présidentiel, on aura noté que les modalités sont du domaine de la première personne du pluriel ainsi que le politique : autour du NOUS, non "pollué " par le rituel, gravitent des volitifs - peu représentés voire absents du voisinage du JE mais aussi des auxiliaires modaux (devons, pouvons) des verbes du constat/bilan ou de 
l'échéance, bref de l'action politique (Nous sommes engagés, nous sommes en train de, nous allons, nous avons). Le JE est au contraire fortement ancré dans le rituel et le métadiscursif. Pourtant, l'analyse de quelques co-présences JE/VOUS a montré que, derrière le rituel, les vœux présidentiels demeurent profondément politiques, et on aura pu entrevoir quelques mécanismes discursifs de persuasion.

\section{BIBLIOGRAPHIE}

Chambre F. (1984). Les vœux présidentiels télévisés sous la cinquième République de 1960 à 1984, Mémoire de DESS en communication politique et sociale, Université Paris I Sorbonne, sous la direction de Jean-Marie Cotteret et de Jacques Gerstlé.

Cotteret J.-M., Moreau R. et al. (1969). Recherches sur le vocabulaire du Général de Gaulle : analyse statistique des allocutions radiodiffusées, 1958-1965. Paris : A. Colin.

Fiala P. \& Leblanc J.-M. (2004). Autour de JE présidentiel. Intervention aux JADT 2004. Louvain- LaNeuve.

Finniss-Boursin F. (1992). Les discours de vœux des présidents de la République, La France au fond des yeux. Paris : Librairie générale de droit et de jurisprudence.

Habert B., Nazarenko A. \& Salem A. (1997). Les linguistiques de corpus. Paris : Armand Colin.

Heiden S. (2002). Manuel Utilisateur de Weblex. Version 4.1, ICAR CNRS/ENS-LSH, <http:// weblex.ens-lsh.fr/doc/ weblex.pdf>.

Labbé D. (1983). François Mitterrand : essai sur le discours. Grenoble : La Pensée sauvage.

Labbé D. (1990). Le vocabulaire de François Mitterrand. Paris : Presse de la Fondation Nationale des Sciences Politiques.

Lafon P. (1984). Dépouillements et statistiques en lexicométrie. Genève-Paris : Slatkine-Champion.

Lamalle C. et al. (2003). Lexico 3, Outils de statistique textuelle. Manuel d'utilisation. Version 3.41 (février 2003), Laboratoire SYLED - CLA2T, Université de la Sorbonne nouvelle Paris 3.

Martinez W. (2003). Contribution à une méthodologie de l'analyse des cooccurrences lexicales multiples dans les corpus textuels, Thèse de Doctorat en Sciences du Langage, Université de la Sorbonne nouvelle - Paris 3, sous la direction d'André Salem.

Mayaffre D. (2004). Paroles de président. Jacques Chirac (1995-2003) et le discours présidentiel sous la Vème République. Paris : Champion.

Rivory E. (1991). Les vœux présidentiels de 1981 à 1990, Mémoire de DESS en communication politique et sociale, Université Paris I Sorbonne, sous la direction de Jean-Marie Cotteret et de Jacques Gerstlé.

Tesnière L. (1959). Eléments de syntaxe structurale. Paris : Klincksieck. 


\section{NOTES}

1.. Mandats de durées différentes, tendances propres à chaque locuteur, différents facteurs peuvent expliquer cette ventilation irrégulière. On comptabilise ainsi pour chaque locuteur un volume lexical différent : De Gaulle (10 discours, 11498 occurrences), Pompidou (5, 2 850), Giscard (7, 6 066), Mitterrand (14, 11 991), Chirac (7, 8720 ).

2.. Environnement thématique (Hyperbase), cooccurrents spécifiques (Lexico3), lexicogrammes simples et récursifs (Weblex), analyse en tri croisé (Alceste).

3.. Ceci tient pour une large part à la nature de l'intervention : le président s'adresse tout naturellement aux Français.

4.. Ceci nous apparaît également au moyen des autres mesures évoquées.

5.. On pourrait parler ici de réduction plutôt que d'une lemmatisation classique.

6.. La forme sais est le deuxième verbe conjugué cooccurrent de la première personne du singulier, juste après le verbe souhaiter, et au cinquième rang des cooccurrents spécifiques du JE.

7.. S'inspirant en partie des travaux de [Lafon 1984] le module informatique Coocs élaboré pour Lexico2 est fondé sur le modèle hypergéométrique et calcule la probabilité de rencontre de deux formes. Pour cela on compare la fréquence globale de chaque cooccurrent $(F)$ avec sa fréquence locale dans un type de contexte défini - ici la phrase - pour déterminer le nombre de rencontres avec le pôle c'est-à-dire la co-

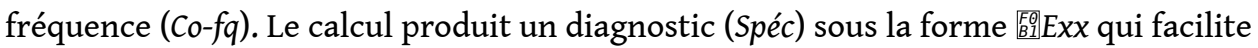
le repérage des cooccurrences les plus spécifiques, positives ou négatives. Cf. [Martinez 2003].

8.. Les résultats présentés ici proviennent d'un traitement qui exclut les mots-outils ce qui a pour effet de réduire la longueur des chemins de cooccurrence explorés. En effet, une comparaison montre qu'en incluant les mots-outils l'analyse rapporte une information qui, en essence, est la même que celle du processus filtré mais qui est polluée par une quantité de bruit très importante. Le traitement filtré rapporte, lui, des chemins de moindre complexité mais à forte teneur informative puisque réduits aux 'mots pleins'.

9.. Le module PolyCoocs pour Lexico2 génère un fichier de données exploitable par le logiciel de dessin Microsoft Visio afin de créer un graphe du réseau cooccurrentiel.

\section{RÉSUMÉS}

Les messages de vœux des présidents de la République constituent un genre discursif particulier, lié à l'épidictique, et fournissent un angle d'approche original pour l'analyse de l'ethos présidentiel vu du rituel politique. A la différence de Cotteret [1969], Labbé [1983, 1990], désormais Mayaffre [2004], nous saisissons le discours présidentiel sous un angle plus étroit mais dont on peut penser qu'il apportera des éléments intéressants si l'on considère cette forme codifiée comme une quintessence du statut présidentiel en représentation. A la suite de Françoise Finniss Boursin [1992] mais aussi de Françoise Chambre [1984] et Elisabeth Rivory [1991], nous nous intéressons aux allocutions de fin d'année des présidents français, mais adoptons une 
démarche plus expérimentale, mobilisant différents outils lexicométriques que nous comparons. Nous saisissons ici l'ethos politique à travers les emplois des marques personnelles inscrivant le locuteur dans son discours, par le biais de plusieurs analyses des cooccurrences.

Enunciative positions in the New Year addresses by the French President during the fifth Republic

The New Year addresses by the French President of the Republic define an original speech genre marked by an epideictic element. They also offer an original approach to presidential ethos from the perspective of political ritual. Unlike Cotteret (1969), Labbé $(1983,1990)$, and now Mayaffre (2004) our analysis studies presidential speech from a narrower angle. We expect however this analysis to yield interesting results when considering this type of coded discourse as the quintessence of the representation of presidential status. In the steps of Françoise Finniss Boursin (1992) and also Françoise Chambre (1984) and Elisabeth Rivory (1991), we have chosen to study New Year addresses by the French President. However our approach is more experimental and mobilizes various lexicometric tools. By applying different cooccurrential methods this work reveals political ethos through the use of personal marks which set the speaker in his discourse.

INDEX

Mots-clés : cooccurrences, poly-cooccurrences, énonciation, rituel politique, ethos

\section{AUTEURS}

JEAN-MARC LEBLANC

CEDITEC, Université Paris12 Val de Marne

WILLIAM MARTINEZ

SYLED, Université Paris 3 Sorbonne Nouvelle 